\title{
The invariant eigen-operator method for Hamiltonians with qubit system- environment models
}

\author{
Yang de Fang \\ Shang rao Radio \& TV University, Shangrao, 334000, China \\ Email:fyd8006@163.com
}

\begin{abstract}
In this paper, we apply the method of "invariant eigen-operator" to study the Hamiltonian of several different qubit-environment models. We find the invariant eigen-operator in terms of these models, which directly leads to the energy-level gap for total system.
\end{abstract}

Keywords: Heisenberg equation, Invariant eigen-operator method, Qubit environment models

\section{Introduction}

Explicit exact solutions and propagators of the Schrödinger equations as well as solutions of the Heisenberg equations of motion were considered and applied to different problems [1]. In general, solving various stationary Schrödinger equations leads to eigenvalues and eigenvectors of dynamic Hamiltonians. Though the Heisenberg equation stands on the same footing as the Schrödinger equation, it is seldom employed for the purpose of directly deriving energy eigenvalues. In a very recent paper [2] author has introduced a new method, i.e. the "invariant eigenoperator" (IEO) method to explore energy-level gap of dynamic Hamiltonians, which is based on both the concept of Schrödinger operator and the Heisenberg equation of motion.

The discovery that quantum algorithms are capable of solving certain types of classically intractable problems has stimulated intensive investigates aimed at the physical implementation of quantum computation. The building block of a quantum computer is called a quantum bit, or simply a qubit, from which multiqubit quantum gates can be constructed and networked to perform any desired quantum logic operation. An ideal qubit is a quantum two-level system whose state can be prepared and controlled by experimenters [3, 4]. The real physical systems of quantum qubit are a two-state system such as photon, electron, atomic nucleus and quantum dot.

A major conceptual as well as technical difficulty in the practical implementation of quantum information processing and quantum control schemes is the unavoidable interaction of quantum systems with their environment. This interaction can destroy quantum superpositions and lead to an irreversible loss of information, a process generally known as decoherence[5]. Theoretical studies of decoherence and dissipation in qubit systems have focused on two canonical system-environment models: (1) a quantum two-level system, represented by a spin$1 / 2$ particle, interacting with a bath of harmonic oscillators (spin-boson model) [6]; and (2) a spin-1/ 2 particle coupled to a bath of other spins (spin-spin model) [7]. Understanding the dynamics of open quantum systems is therefore of considerable importance. In order to control and 
operate qubit accurately, and realize quantum communication and quantum computation, the study of qubit systems' energy level( or energy level gap ) and the influence of environment to qubit systems' energy becomes one of the important mission of quantum informatics. In this manuscript, we apply the method of "invariant eigen-operator" to study in different qubit-environment models, the influence to qubit system energy level.

\section{The invariant eigen-operator method}

In quantum mechanics, the discrete energy levels and simultaneously the eigenstates of dynamic systems are usually derived by solving the appropriate Schrödinger

equation $i(d / d t) \psi=\hat{H} \psi$ (in thia paper, we assume $\hbar=1$ for simplicity). However, owing to the Heisenberg equation of motion,

$$
i \frac{d}{d t} \hat{Q}=[\hat{Q}, \hat{H}]
$$

which is of the same form and importance as the Schrödinger equation. Since Eq.(1) does not involve wavefunctions or eigenvectors, it can hardly be straighforwardly employed to derive energy-level formulas. In Ref. [2], the authors reported that the Heisenberg equation of motion can also be used to deduce the energy-level gap of certain dynamic systems if one can find some appropriate eigen-operators $\hat{Q}$ of the square of the Schrödinger operator $i(d / d t)$. Its main idea is as follows. For the certain quantum system, there is $\hat{Q}$ satisfying the following eigenvectorlike equation

$$
\left(i \frac{d}{d t}\right)^{n} \hat{Q}=[\ldots[[\hat{Q}, \hat{H}], \hat{H}] \ldots]=G \hat{Q}
$$

where $G$ is real. We can judge that $\sqrt[n]{G}$ is the energy-level gap between two adjacent energy levels of the dynamic Hamiltonian $\hat{H}$.

To clarify this point of view, we take $n=2$ in Eq.(2), and assuming that that $|m\rangle$ and $|n\rangle$ are two adjacent different stationary eigen-states of Hamiltonian $\hat{H}$ with eigen-values $E_{m}$ and $E_{n}$, respectively. Using the Heisenberg equation, we obtain

$$
\begin{aligned}
& \left\langle m\left|\left(i \hbar \frac{d}{d t}\right)^{2} \hat{Q}\right| n\right\rangle=\left\langle m\left|\left[\left[\hat{Q}, \hat{H}_{0}\right], \hat{H}_{0}\right]\right| n\right\rangle \\
& =\left(E_{m}-E_{n}\right)^{2}\langle m|\hat{Q}| n\rangle \\
& =G^{2}\langle m|\hat{Q}| n\rangle
\end{aligned}
$$

where

$\boldsymbol{G}=\left|E_{m}-E_{n}\right|$,

whenever $\langle m|\hat{Q}| n\rangle$ is nonzero matrix element, the energy gap between $|m\rangle$ and $|n\rangle$ can be obtained as $G=\left|E_{m}-E_{n}\right|$. Thus, this new method is named as the invariant eigen-operator method.

\section{The invariant eigen-operator method for Some Hamiltonians}

It well known set of canonical models describes the environment as a set of uncoupled oscillators-these include the 'spin-boson' model and the 'CaldeiraLeggett' model. The spin-boson model couples a central two-level system to the oscillators, and is thus the analogue of the central spin model. These oscillator models all derive from a scheme proposed by Feynman and Vernon. Oscillator models are thus best adapted to delocalized environmental modes. The simplest Hamiltonian of the spin-boson model is written as[8]

$\hat{H}_{1}=-\frac{1}{2} \varepsilon \hat{\sigma}_{z}+\sum_{j=1}\left(\frac{1}{2 m_{j}} \hat{p}_{j}^{2}+\frac{1}{2} m_{j} \omega_{j}^{2} \hat{x}_{j}^{2}\right)-\hat{\sigma}_{z} \sum_{j=1} \alpha_{j} \hat{x}_{j}$

In the spin-boson model, another Hamiltonian of the spin-boson model is 
$\hat{H}_{2}=-\frac{1}{2} \varepsilon \hat{\sigma}_{z}+\sum_{j=1}\left(\frac{1}{2 m_{j}} \hat{p}_{j}^{2}+\frac{1}{2} m_{j} \omega_{j}^{2} \hat{x}_{j}^{2}\right)-\frac{1}{2} \hat{\sigma}_{z} \sum_{j=1} \beta_{j} \hat{x}_{j}^{2}$

The quantum dynamics of quantum systems is always complicated by their coupling to many environmental modes. At low temperature these environmental effects are dominated by localized modes. This environment, at low energies, maps onto a 'spin bath' model. This contrasts with 'oscillator bath' models which describe delocalized environmental modes such as electrons, phonons, photons, magnons, etc. The simplest Hamiltonian of the spin-spin model is written as [9]

$$
\hat{H}_{3}=\frac{1}{2} \omega \hat{\sigma}_{z}+\sum_{j=1} \frac{1}{2} \Omega_{j} \hat{\sigma}_{j z}+\frac{1}{2} \hat{\sigma}_{z} \sum_{j=1} \gamma_{j} \hat{\sigma}_{j z}
$$

In this section we shall apply the invariant eigen-operator method to derive the energy gap of the qubit systemenvironment models.

Case 1 According to the invariant eigen-operator method, firstly we should consider the basic commutative relations using the Heisenberg equation

$$
\begin{gathered}
{\left[\hat{x}_{j}, \hat{H}_{1}\right]=\frac{1}{m_{j}} \hat{p}_{j}} \\
{\left[\hat{p}_{j}, \hat{H}_{1}\right]=-\left(m_{j} \omega_{j}^{2} \hat{x}_{j}-\alpha_{j} \hat{\sigma}_{z}\right)} \\
{\left[\hat{\sigma}_{z} \hat{x}_{j}, \hat{H}_{1}\right]=\frac{1}{m_{j}} \hat{\sigma}_{z} \hat{p}_{j}} \\
{\left[\hat{\sigma}_{z} \hat{p}_{j}, \hat{H}_{1}\right]=-\left(m_{j} \omega_{j}^{2} \hat{\sigma}_{z} \hat{x}_{j}-\alpha_{j}\right)} \\
{\left[\hat{\sigma}_{z}, \hat{H}_{1}\right]=0}
\end{gathered}
$$

Based on Hamiltonian $\hat{H}_{1}$ in Eq.(3), we suppose that the invariant eigen-operator in the case is

$$
\hat{Q}_{1}=g_{1} \hat{x}_{j}+g_{2} \hat{p}_{2}+g_{3} \hat{\sigma}_{z} \hat{x}_{j}+g_{4} \hat{\sigma}_{z} \hat{p}_{j}+g_{5} \hat{\sigma}_{z}
$$

Substituting Eq. (9) into Eq. (2), combing Eqs. (3) (7), we have

$$
\begin{gathered}
{\left[\hat{Q}_{1}, \hat{H}_{1}\right]=-m_{j} \omega_{j}^{2} g_{2} \hat{x}_{j}+\frac{1}{m_{j}} g_{1} \hat{p}_{j}-m_{j} \omega_{j}^{2} g_{4} \hat{\sigma}_{z} \hat{x}_{j}} \\
+\frac{1}{m_{j}} g_{3} \hat{\sigma}_{z} \hat{p}_{j}+\alpha_{j}\left(g_{2}+g_{4}\right) \hat{\sigma}_{z}
\end{gathered}
$$

If the operator $\hat{Q}_{1}$ satisfies Eq. (2), while $n=1$, comparing Eq. (8) and Eq. (10), we obtain

$$
\left(\begin{array}{ccccc}
0 & -m_{j} \omega_{j}^{2} & 0 & 0 & 0 \\
m_{j}^{-1} & 0 & 0 & 0 & 0 \\
0 & 0 & 0 & -m_{j} \omega_{j}^{2} & 0 \\
0 & 0 & m_{j}^{-1} & 0 & 0 \\
0 & \alpha_{j} & 0 & \alpha_{j} & 0
\end{array}\right)\left(\begin{array}{l}
g_{1} \\
g_{2} \\
g_{3} \\
g_{4} \\
g_{5}
\end{array}\right)=G_{1}\left(\begin{array}{l}
g_{1} \\
g_{2} \\
g_{3} \\
g_{4} \\
g_{5}
\end{array}\right)
$$

Eq.(11)'s eigenvalues are: $\pm \omega_{j}, 0$. Thus the energy-level gap of Hamiltonian $\hat{H}_{1}$ is

$$
G_{1}=\omega_{j}
$$

As the coupled quantum system of the the spin-boson model is described by Eq.(3), we can easily see that the energy level gap of quantum system is given by $G_{1}$.

Case 2 We can list some basic commutative relations using the Heisenberg equation

$$
\begin{aligned}
& {\left[\hat{x}_{j}, \hat{H}_{2}\right]=\frac{1}{m_{j}} \hat{p}_{j}} \\
& {\left[\hat{p}_{j}, \hat{H}_{2}\right]=-\left(m_{j} \omega_{j}^{2} \hat{x}_{j}-\beta_{j} \hat{\sigma}_{z} \hat{x}_{j}\right)} \\
& {\left[\hat{\sigma}_{z} \hat{x}_{j}, \hat{H}_{2}\right]=\frac{1}{m_{j}} \hat{\sigma}_{z} \hat{p}_{j}} \\
& {\left[\hat{\sigma}_{z} \hat{p}_{j}, \hat{H}_{2}\right]=-\left(m_{j} \omega_{j}^{2} \hat{\sigma}_{z} \hat{x}_{j}-\beta_{j} \hat{x}_{j}\right)}
\end{aligned}
$$

The parameter $g_{j}$ is to be determined. 
From Eq.s (12) (15) we guess that the invariant eigen-operator of $\hat{H}_{2}$ is

$\hat{Q}_{2}=h_{1} \hat{x}_{j}+h_{2} \hat{p}_{2}+h_{3} \hat{\sigma}_{z} \hat{x}_{j}+h_{4} \hat{\sigma}_{z} \hat{p}_{j}$

The parameters $h_{j}$ are to be determined.

Substituting Eq. (15) into Eq. (2), combing Eq.s (12) (15), we have

$$
\begin{array}{r}
{\left[\hat{Q}_{2}, \hat{H}_{2}\right]=\left(-m_{j} \omega_{j}^{2} h_{2}+\beta_{j} h_{4}\right) \hat{x}_{j}+\frac{1}{m_{j}} h_{1} \hat{p}_{j}} \\
+\left(\beta_{j} h_{2}-m_{j} \omega_{j}^{2} h_{4}\right) \hat{\sigma}_{z} \hat{x}_{j}+\frac{1}{m_{j}} h_{3} \hat{\sigma}_{z} \hat{p}_{j}
\end{array}
$$

Comparing Eq. (16) with Eq. (2), it is necessary to ask for $(n=1)$

$$
\left(\begin{array}{cccc}
0 & -m_{j} \omega_{j}^{2} & 0 & \beta_{j} \\
m_{j}^{-1} & 0 & 0 & 0 \\
0 & \beta_{j} & 0 & -m_{j} \omega_{j}^{2} \\
0 & 0 & m_{j}^{-1} & 0
\end{array}\right)\left(\begin{array}{l}
h_{1} \\
h_{2} \\
h_{3} \\
h_{4}
\end{array}\right)=G_{2}\left(\begin{array}{l}
h_{1} \\
h_{2} \\
h_{3} \\
h_{4}
\end{array}\right)
$$

Eq.(18)'s eigenvalues are: $\sqrt{\omega_{j}^{2} \pm \beta_{j} / m_{j}}$. Thus the energy-level gap of Hamiltonian $\hat{H}_{2}$ also has two kinds of probabilities.

$$
G_{2}=\sqrt{\omega_{j}^{2} \pm \frac{\beta_{j}}{m_{j}}}
$$

Thus, the coupled quantum system of the the spin-boson model is described by Eq.(12), we can easily see that the energy level gap of quantum system is given by $G_{2}$.

In brief, we can easily know that: the energy level gap of quantum system is closely related to the interaction form of qubit and environment. The energy level gap is determined by both the inherent parameters of the oscillator models and coupling parameter. Therefore, in order to control and operate qubit accurately, realize quantum communication and quantum computation, we need study the interaction form of qubit and environment deeply.

Case 3 The Hamiltonian of the spinspin model is written as

The study of spin system can be described by a boson operator. Schwinger boson is a kind of boson operator describing spin system[10]. Set

$$
\sigma_{j z}=\frac{1}{2}\left(a_{j}^{+} a_{j}-b_{j}^{+} b_{j}\right)
$$

Then, the Hamiltonian $\hat{H}_{3}$ is rewritten as

$$
\begin{aligned}
\hat{H}_{3}= & \frac{1}{2} \omega \sigma_{z}+\sum_{j=1} \frac{1}{4} \Omega_{j}\left(a_{j}^{+} a_{j}-b_{j}^{+} b_{j}\right) \\
& +\frac{1}{4} \sigma_{z} \sum_{j=1} \gamma_{j}\left(a_{j}^{+} a_{j}-b_{j}^{+} b_{j}\right)
\end{aligned}
$$

Using the basic commutative relations of operators, we have

$\left[a_{j}, H_{3}\right]=\frac{1}{4} \Omega_{j} a_{j}+\frac{1}{4} \gamma_{j} \sigma_{z} a_{j}$

$\left[a_{j}^{+}, H_{3}\right]=-\frac{1}{4} \Omega_{j} a_{j}^{+}-\frac{1}{4} \gamma_{j} \sigma_{z} a_{j}^{+}$

$\left[b_{j}, H_{3}\right]=-\frac{1}{4} \Omega_{j} b_{j}-\frac{1}{4} \gamma_{j} \sigma_{z} b_{j}$

$\left[b_{j}^{+}, H_{3}\right]=\frac{1}{4} \Omega_{j} b_{j}^{+}+\frac{1}{4} \gamma_{j} \sigma_{z} b_{j}^{+}$

From Eq.s (25) (28) we guess that the invariant eigen-operator of $\hat{H}_{3}$ is

$$
\begin{aligned}
\hat{Q}_{3} & =\left(q_{1}+q_{5} \hat{\sigma}_{z}\right) \hat{a}_{j}+\left(q_{2}+q_{6} \hat{\sigma}_{z}\right) \hat{a}_{j}^{+} \\
& +\left(q_{3}+q_{7} \hat{\sigma}_{z}\right) \hat{b}_{j}+\left(q_{4}+q_{8} \hat{\sigma}_{z}\right) \hat{b}_{j}^{+}
\end{aligned}
$$

The parameters $q_{j}$ are to be determined. If the operator $\hat{Q}_{3}$ is the invariant eigenoperator for $\hat{H}_{3}, q_{j}$ must satisfies the equation following 


$\left(\begin{array}{cccccccc}\Omega_{j} & 0 & 0 & 0 & \gamma_{j} & 0 & 0 & 0 \\ 0 & -\Omega_{j} & 0 & 0 & 0 & -\gamma_{j} & 0 & 0 \\ 0 & 0 & -\Omega_{j} & 0 & 0 & 0 & -\gamma_{j} & 0 \\ 0 & 0 & 0 & \Omega_{j} & 0 & 0 & 0 & \gamma_{j} \\ \gamma_{j} & 0 & 0 & 0 & \Omega_{j} & 0 & 0 & 0 \\ 0 & -\gamma_{j} & 0 & 0 & 0 & -\Omega_{j} & 0 & 0 \\ 0 & 0 & -\gamma_{j} & 0 & 0 & 0 & -\Omega_{j} & 0 \\ 0 & 0 & 0 & \gamma_{j} & 0 & 0 & 0 & \Omega_{j}\end{array}\right)\left(\begin{array}{c}q_{1} \\ q_{2} \\ q_{3} \\ q_{4} \\ q_{5} \\ q_{6} \\ q_{7} \\ q_{8}\end{array}\right)=4 \mathrm{G}_{3}\left(\begin{array}{c}q_{1} \\ q_{2} \\ q_{3} \\ q_{4} \\ q_{5} \\ q_{6} \\ q_{7} \\ q_{8}\end{array}\right)$

Eq.(27)'s eigenvalues are: $\left(\Omega_{j} \pm \gamma_{j}\right) / 4$, $-\left(\Omega_{j} \pm \gamma_{j}\right) / 4$. Thus the energy-level gap of Hamiltonian $\hat{H}_{3}$ also has two kinds of probabilities.

$$
G_{3}=\frac{1}{4}\left(\Omega_{j} \pm \gamma_{j}\right)
$$

In above calculations, we get a system of linear homogeneous equations though calculating the first order eigen operator equation $\left[\hat{Q}_{j}, \hat{H}\right]=G_{j} \hat{Q}_{j}$, and then get the energy-level gap of the systems though calculating the eigenvalue of system of linear homogeneous equations. In fact, we also can get the energy-level gap of the systems though calculating the second order eigen operator equation [ $\left.\left[\hat{Q}_{j}, \hat{H}\right], \hat{H}\right]=G_{j} \hat{Q}_{j}$, and comparing the undetermined coefficients.

\section{Conclusions}

In summary, we have adopted the invariant eigen-operator method to tackle in different qubit-environment models, the influence for the energy level of qubit system. Because energy transition directly correspond to energy gaps, so our result has an important guidance meaning for achieving the exact changing and controlling to the quanta bit in the laboratory. This approach seems concise and direct and can be extended to tackle other Hamiltonian models.

\section{Reference}

[1]. Michelot F.: Solution for an arbitrary number of coupled identical oscillators. Phys. Rev. A 45, 4271(1992).
[2]. Fan H. Y. and Li C.: Invariant eigenoperator of the square of Schrodinger operator for deriving energy-level gap. Phys. Lett. A 321, 75 (2004).

[3] Yang C. P. and Han S., Rotation gate for a three-level superconducting quantum interference device qubit with resonant interaction, Phys. Rev. A 74, 044302(2006).

[4] Yu Y., Han S., Chu X. et al., Coherent Temporal Oscillations of Macroscopic Quantum States in a Josephson Junction, Science 296, 889 (2002).

[5] Tian L., LIoyd S. and Orlando T. P., Decoherence and relaxation of a superconducting quantum bit during measurement, Phys. Rev. B. 65, 144516(2002)

[6] Leggett, A.J., Chakravarty, S., Dorsey, A.T., et al.: Dynamics of the dissipative two-state system. Rev. Mod. Phys. 59, 1 (1987)

[7] Prokof'ev N. V. and Stamp P. C. E., Theory of the spin bath, Rep. Prog. Phys. 63, 669 (2000).

[8] Hartmann L., Goychuk I., Grifoni M., Hanggi P., Driven tunneling dynamics: Bloch-Redfield theory versus path-integral approach, Phys. Rev. E. 61, R4687(2000).

[9] Krovi H., Oreshkov O., Ryazanov M., Lidar D. A., Non-Markovian dynamics of a qubit coupled to an Ising spin bath, Phys. Rev. A. 76, 052117(2007).

[10] Wei C G, Tao R B, Schwnger-boson mean-field theory of the twodimensional antiferromagnetic Heisenberg model with a square lattice, Phys. Rev. B. 50, 6840(1994). 